\title{
Combination of Transverse Mode Selection and Active Longitudinal Mode-Locking of Broad Area Semiconductor Lasers
}

\author{
Christoph Doering and Henning Fouckhardt \\ Integrated Optoelectronics and Microoptics Research Group, Physics Department, Kaiserslautern University of Technology, \\ P.O. Box 3049, D-67653 Kaiserslautern, Germany \\ Correspondence should be addressed to Christoph Doering; cdoering@physik.uni-kl.de
}

Received 21 February 2014; Accepted 30 June 2014; Published 13 July 2014

Academic Editor: Michele Norgia

Copyright (C) 2014 C. Doering and H. Fouckhardt. This is an open access article distributed under the Creative Commons Attribution License, which permits unrestricted use, distribution, and reproduction in any medium, provided the original work is properly cited.

\begin{abstract}
Experimental results of the combination of transverse mode selection and active mode-locking with anti-reflection-coated broad area lasers (BALs) are presented. The BALs are subject to feedback from a free-space external Fourier-optical $4 f$-setup with a reflective spatial frequency filter in the Fourier-plane for transverse mode selection. Driving the BALs with a high frequency modulated pump current above threshold active longitudinal mode-locking is achieved. Pulse durations as low as 88 ps are obtained, while the Gaussian-like fundamental or a higher order transverse mode up to mode number 5 is selected on purpose. Pulse duration and shape are nearly independent of the selected transverse mode.
\end{abstract}

\section{Introduction}

Semiconductor lasers are widely used in many of today's photonic applications, due to their advantages like reliability, small size, the opportunity for electrical pumping, and an excellent efficiency. However, catastrophic optical mirror damage (COMD) of the edge-emitting laser facets limits the output power of laser diodes. The use of wide active stripes in so-called broad-area lasers (BALs) circumvents this restriction. BALs with an emitter width of $50 \mu \mathrm{m}$ up to $1 \mathrm{~mm}$ [1] are available. The large size of the active region and the high power lead to strong nonlinear optical coupling of the amplified wave and the active material and result in an uncontrolled temporally unstable emission of more than one transverse mode. The emission of BALs can be controlled by, for example, using a grating integrated in the semiconductor chip [2-4] or by external cavities [5-10]. The latter also influence the longitudinal mode emission and the stability of the laser. On the other hand feedback from external cavities can also destabilize the emission of laser diodes [11-15] leading to selfpulsation.

Semiconductor lasers are advantageous for the generation of short optical pulses due to the large gain bandwidth of
$30-45 \mathrm{~nm}$. Mode-locking is the most common method applied to generate short pulses in semiconductor lasers. Two mode-locking techniques can be distinguished, that is, active and passive mode-locking [16]. In active mode-locking intracavity amplitude or phase modulators are used to force coupling of the longitudinal cavity modes. The advantage of active over passive mode-locking is a higher reproducibility of pulses with respect to pulse height and width [16]. Pulse widths in the picosecond range are reported by many groups investigating active or passive mode-locking of external cavity narrow stripe laser diodes [17-20]. However the power of available narrow stripe diodes is limited by COMD as mentioned above. Higher pulse powers can be extracted from mode-locked BALs, even though high frequency modulation is difficult due to the large capacitance. Passively modelocked BALs can emit pulses with duration of 15 ps [21], without any attention to transverse mode emission of the BAL. The generation of 13 ps pulses by injection of an initial short optical pulse into a BAL is shown in [22]. Even mode locking of transversal modes in BALs is investigated leading to spatially alternating emission with a repetition rate of around $3.4 \mathrm{GHz}$ [23]. 
In this paper we present experimental results of active mode-locking of BAL (anti-reflection- (AR-) coated on one facet) being subject to feedback from a free-space external Fourier-optical $4 f$-cavity with a reflective spatial frequency filter in the Fourier-plane for transverse mode selection (i.e., $2 f$ plus the way back after reflection from a reflective spatial filter). Active mode-locking with a repetition rate of about $1 \mathrm{GHz}$ is achieved through the modulation of the laser pump current for $\mathrm{a} \approx 150 \mathrm{~mm}$ long external cavity.

\section{Experimental Work}

The experiments are performed using a BAL highly ARcoated on the front facet (that one directed towards the external cavity) with a residual reflectivity of $R_{2}<8 \cdot 10^{-5}$ in the $4 f$-set-up depicted in Figure 1. The laser has an emission wavelength of $\lambda=800 \mathrm{~nm}$. The external cavity consists of a biconvex lens $\mathrm{L}_{1}$, with a focus length of $f_{1}=75 \mathrm{~mm}$. The front facet and a plane mirror $M$ are placed in the front and in the rear focal plane of the lens $\mathrm{L}_{1}$, respectively. A slit SL movable in the $x$-direction in front of the mirror forms the spatial frequency filter for transverse mode selection. To favour the fundamental transverse mode (mode number 0 ) the slit has to be centered around the optical axis. The slit width $s$ of the spatial frequency filter is set to $s=970 \mu \mathrm{m}$, to select a single transverse mode [10]. Active mode-locking is achieved by driving the BAL with a sinusoidal radio frequency (RF) signal from a high power signal generator (Rohde \& Schwarz, SMGL). A bias tee (Picosecond Pulse Labs, 5580) is inserted to combine the RF current modulation and the laser dc pump current.

The emitted modulated BAL intensity is measured at the rear facet with an ultrafast photodiode (Hama-matsu G4176 used together with a bias tee Picosecond Pulse Labs 5541A) connected to a $50 \mathrm{GHz}$ sampling oscilloscope (Tektronix, CSA 803A). The spatial filter is aligned onto the optical axis for fundamental transverse mode selection. Shifting the filter a distance $d$ perpendicularly away from the optical axis a certain (higher) transverse mode can be selected [10].

\section{Experimental Results of Simultaneous Mode-Locking and Transverse Mode Selection}

Active mode-locking is achieved by pump current modulation here. The maximum power of the RF generator is $36 \mathrm{dBm}$, corresponding to a maximum ac pump current of $I_{\mathrm{ac} \text {, max }}=0.57 \cdot I_{\mathrm{th}}$ nominal. The power reaching the BAL is about $20 \%$ lower due to an electrical impedance mismatch between the BAL and the RF generator. Therefore, only $I_{\mathrm{ac}}=$ $80 \% \cdot I_{\mathrm{ac} \text {, max }}=0.46 \cdot I_{\mathrm{th}}$ is applicable. To achieve pulsed pump currents of at least $\approx 2 \cdot I_{\text {th }}$, the dc part of the pump current has to be significantly higher than $I_{\text {th }}$, in order not to fall below $I_{\text {th }}$ in any part of the pump cycle.

Pulse width and intensity are strongly depending on the ratio of the dc and the ac part of the pump current. Considering the fixed ac part $I_{\mathrm{ac}}=80 \% \cdot I_{\mathrm{ac}, \max }=0.46 \cdot I_{\mathrm{th}}$ this means a variation of the $\mathrm{dc}$ part only. Thus Figure 2 shows the measured pulse widths in dependence of the dc pump current. The minimum pulse width is obtained at a dc pump

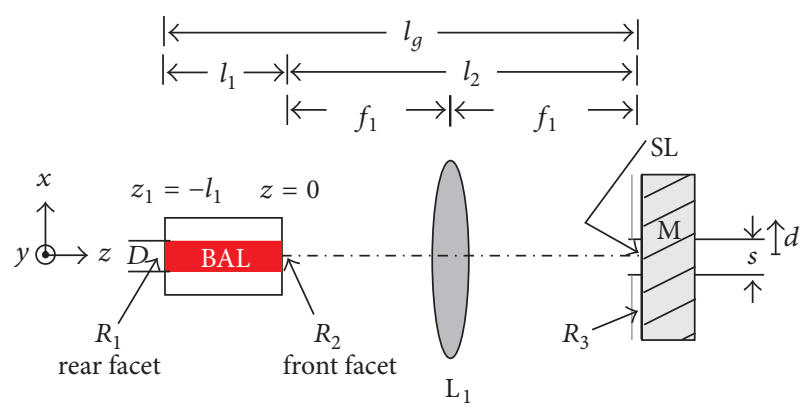

FIgURE 1: Top view of the reflective $4 f$-setup ( $2 f$ plus $2 f$ after reflection) with a spatial frequency filter (slit in front of a mirror); BAL with an emission wavelength of $\lambda=800 \mathrm{~nm}$, SL: slit, $s$ : adjustable slit width, $\mathrm{L}_{1}$ : lens, $l_{1}=2100 \mu \mathrm{m}$ : length of BAL, $l_{2}$ : external cavity length, $l_{g}=l_{1}+l_{2}$ : total length, $D$ : emitter width, and $R_{1}, R_{2}$, and $R_{3}$ : reflectivities of the rear facet, the front facet, and the mirror $M$, respectively.

current of $I_{\mathrm{dc}} \approx 1.48 \cdot I_{\mathrm{th}}$ (giving a minimum current of $I_{\min } \approx$ $1.02 \cdot I_{\text {th }}$ during the pump cycle). And the dependence of the pulse intensity on the dc pump current is given in Figure 3. The maximum pulse intensity is also achieved at the same $\mathrm{dc}$ pump current of $I_{\mathrm{dc}} \approx 1.48 \cdot I_{\mathrm{th}}$. At higher dc parts the pulse intensity saturates, while the pulse duration is increasing. The result can be understood in view of the sinusoidal ac pump current part; that is, for the optimum of $I_{\mathrm{dc}} \approx 1.48 \cdot I_{\mathrm{th}}$ (@ $\left.I_{\mathrm{ac}}=0.46 \cdot I_{\mathrm{th}}\right)$ the pump current never gets below threshold (even in the negative half cycle of the sinusoidal modulation). At higher dc current parts the solitary laser diode takes over and the influence of the external resonator decreases. This way the mode-locking gets gradually weaker, resulting in an increase of the pulse width.

Choosing the optimum dc pump current of $I_{\mathrm{dc}}=$ $820 \mathrm{~mA} \approx 1.48 \cdot I_{\text {th }}\left(I_{\text {th }}\right.$ is always considered in the case with feedback from the external cavity) the temporal sequence of emerging mode-locked pulses is investigated. The results are given in Figure 4. The duration of the pulses (full width at half maximum, FWHM) is 88 ps. The time interval between two subsequent pulses comes to 1004 ps, corresponding to an applied modulation frequency of $996 \mathrm{MHz}$ due to an external cavity length slightly larger than $150 \mathrm{~mm}$. The red line in Figure 4 shows a sech ${ }^{2}$ fit to the measured data. These $s e c h^{2}-$ shaped pulses are obtained, while the BAL is operating on the Gaussian-like fundamental transverse mode.

The power-spectrum of the BAL in the continuous wave (cw) mode and in a mode-locked state is measured with a high resolution fiber-optic spectrometer (Avantes, AvaSpec3648). The results of two measurements are illustrated in Figure 5.

The bandwidth of the longitudinal mode distribution above threshold (thick black line) of the BAL in a modelocked state is about $0.05 \mathrm{~nm}$, whereas it is $0.78 \mathrm{~nm}$ with $\mathrm{cw}$ operation (thin red line). The average power at the rear facet for $\mathrm{cw}$ operation is measured to be $620 \mathrm{~mW}$ by a power meter (Melles Griot, 13PDC).

Shifting the spatial frequency filter unit away from the optical axis (in $x$-direction, see Figure 1) causes an increase of 


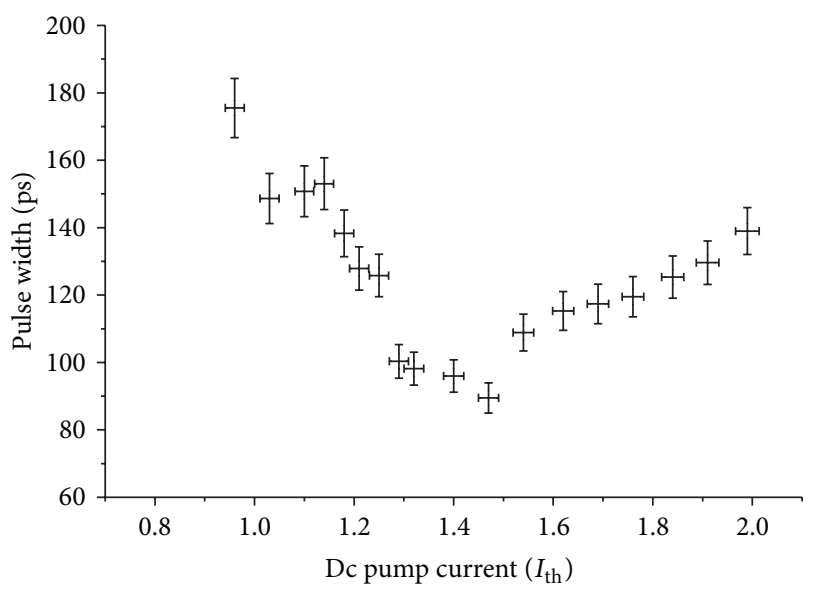

FIgURE 2: Measured pulse width at different dc pump currents and a fixed ac pump current of $I_{\mathrm{ac}}=0.46 \cdot I_{\mathrm{th}}$. The BAL is running on the fundamental transverse mode.

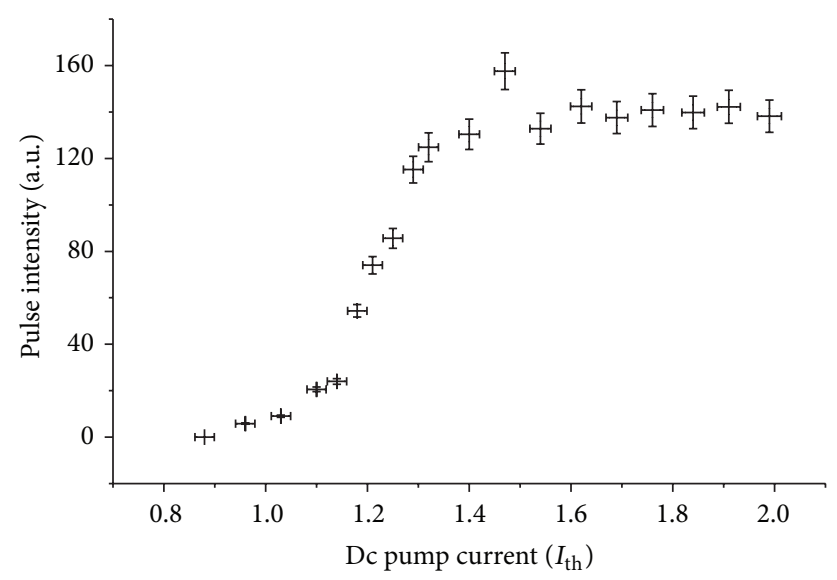

FIgURE 3: Measured pulse intensity at different dc pump currents and a fixed ac pump current of $I_{\mathrm{ac}} \approx 0.46 \cdot I_{\mathrm{th}}$.

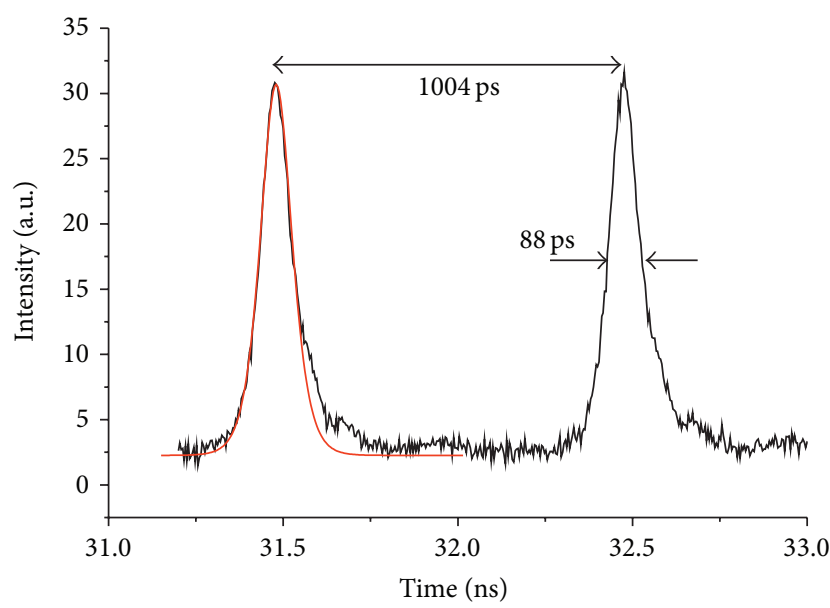

FIGURE 4: Temporal sequence of mode-locked pulses (black line), while the BAL is running on the fundamental transverse mode. Pulses with an FWHM of as low as 88 ps emerge. The interval of 1004 ps correlates to a modulation frequency of $996 \mathrm{MHz}$. A sech ${ }^{2}$ fit to the measured data is also given (red line).

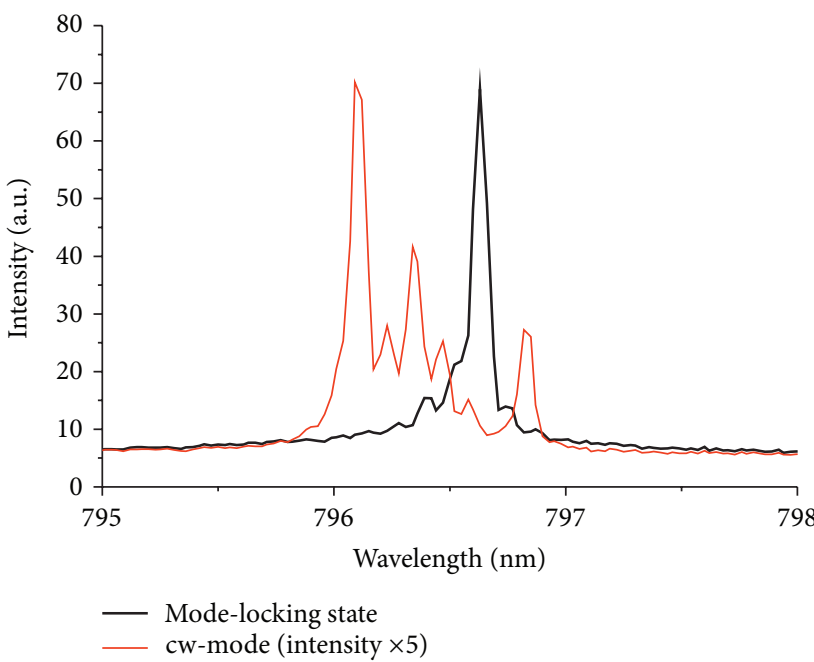

FIGURE 5: Measured power spectra of the BAL operating $\mathrm{cw}$ (thin red line) or in the mode-locked state (thick black line). The spatial frequency filter is aligned onto the optical axis for selection of the fundamental transverse mode here.

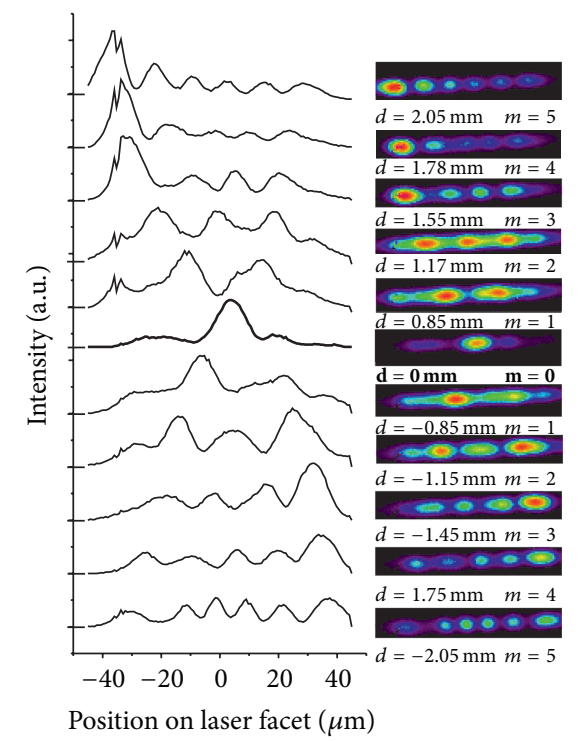

FIGURE 6: CCD camera images of the near-field intensity patterns (right) and line scans of these patterns at different filter positions $d$. The Gaussian-like fundamental mode number $0(m=0)$ and higher order transverse modes up to mode number $m=5$ can be selectively excited.

the number of intensity maxima in the near-field distribution emitted by the BAL, as it is obvious from the results in Figure 6 . The figure shows CCD camera images (right) and line scans of the near-field intensity patterns. Higher order transverse modes up to $m=5$ can be selectively excited with increasing distance $d$ of the center of the spatial frequency filter from the optical axis, while the laser is in the mode-locked state (now $I_{\mathrm{dc}}=1.57 \cdot I_{\mathrm{th}}$ and $\left.I_{\mathrm{ac}}=0.46 \cdot I_{\mathrm{th}}\right)$. 


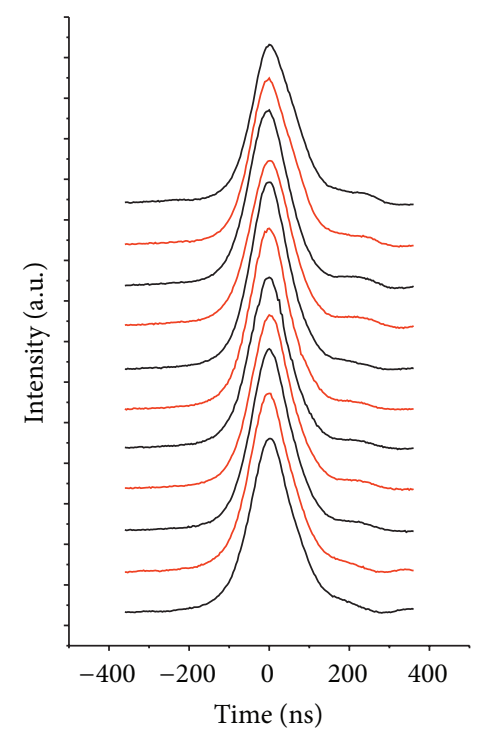

Figure 7: Measured temporal pulse shapes for different distances $d$ of the filter slit to the optical axis. The shape of the pulses, their width, and their intensity are nearly independent of the slit displacement and the transverse mode number.

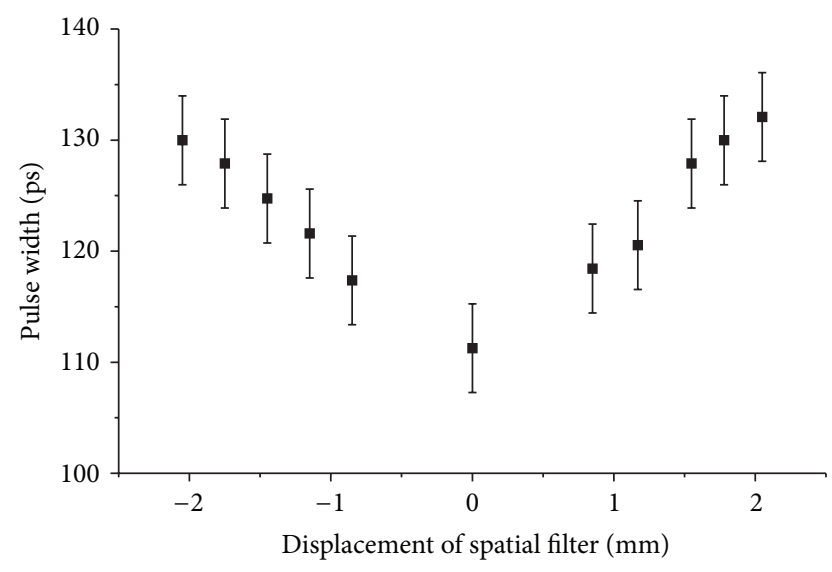

FIgURE 8: Measured pulse width (FWHM) - using a different BAL sample as compared to the case of Figure 4-for different distances $d$ of the spatial frequency filter slit to the optical axis. The pulse duration increases nearly linearly by about $3.2 \%$ with each increment of the transverse mode number $m$.

The displacement $d$ of the spatial filter away from the optical axis, necessary to excite a certain transverse mode, is determined by

$$
d=\frac{m+1}{2} \frac{\lambda f_{1}}{D}
$$

where the mode with transverse mode number $m(m=$ $0,1,2,3, \ldots)$ has $m+1$ intensity maxima in the near-field and $D=100 \mu \mathrm{m}$ is the emitter width.

Due to the asymmetric filtering (i.e., the slit can only be placed on one side of the optical axis at any moment) the intensity patterns of each transverse mode are also asymmetric [10]. Figure 7 shows the measured temporal pulse shapes at different distances $d$ of the slit from the optical axis - that is, a different selected order $m$ of the transverse mode. Obviously the $s e h^{2}$-like pulse shape remains unchanged. But the pulse width increases linearly by only about $3.2 \%$ with each order (increment 1) of the transverse mode, as illustrated in Figure 8 , which was extracted from experiments with a different BAL sample, giving pulse widths of $\approx 120$ ps.

\section{Conclusions}

Radio frequency (RF) modulation at about $1 \mathrm{GHz}$ of the pump current of a broad-area laser (BAL) with an external $4 f$ cavity (acting as a spatial frequency filter unit) causes active mode-locking with a pulse width of as low as $88 \mathrm{ps}$. The selection of a higher order transverse mode has no influence on the temporal $\operatorname{sech}^{2}$-like shape and only a weak influence on the width and intensity of the mode-locked pulses. Pulse duration increases linearly by only $3.2 \%$ with each increment of the number of the selected transverse modes. This indicates that coherent-optical transverse mode selection has only a negligible effect on longitudinal mode-locking. Hence, transverse mode selection and active longitudinal modelocking of BALs are possible simultaneously.

\section{Conflict of Interests}

The authors declare that there is no conflict of interests regarding the publication of this paper.

\section{Acknowledgment}

This research was partially funded by the German Research Foundation (Deutsche Forschungsgemeinschaft, DFG) under contract Fo 157/19.

\section{References}

[1] M. Chi, B. Thestrup, and P. M. Petersen, "Self-injection locking of an extraordinarily wide broad-area diode laser with a 1000$\mu \mathrm{m}$-wide emitter," Optics Letters, vol. 30, pp. 1147-1149, 2005.

[2] D. Mehuys, S. O'Brien, R. J. Lang, A. Hardy, and D. F. Welch, " $5 \mathrm{~W}$, diffraction-limited, tapered-stripe unstable resonator semiconductor laser," Electronics Letters, vol. 30, no. 22, pp. 1855-1856, 1994.

[3] S. R. Šehnić, G. A. Evans, T. M. Chou et al., "Single frequency 1550-nm AlGaInAs-InP tapered high-power laser with a distributed Bragg reflector," IEEE Photonics Technology Letters, vol. 14, no. 7, pp. 890-892, 2002.

[4] O. K. Kwon, K. H. Kim, E. D. Sim, J. H. Kim, and K. R. $\mathrm{Oh}$, "Monolithically integrated multiwavelength grating cavity laser," IEEE Photonics Technology Letters, vol. 17, no. 9, pp. 17881790, 2005.

[5] Y. Champagne, S. Mailhot, and N. McCarthy, "Numerical procedure for the lateral-mode analysis of broad-area semiconductor lasers with an external cavity," IEEE Journal of Quantum Electronics, vol. 31, no. 5, pp. 795-810, 1995.

[6] C. Simmendinger, M. Münkel, and O. Hess, "Controlling complex temporal and spatio-temporal dynamics in semiconductor lasers," Chaos, Solitons and Fractals, vol. 10, no. 4, pp. 851-864, 1999. 
[7] C. Simmendinger, D. Preißer, and O. Hess, "Stabilization of chaotic spatiotemporal filamentation in large broad area lasers by spatially structured optical feedback," Optics Express, vol. 5, no. 3, pp. 48-54, 1999.

[8] N. McCarthy, S. Mailhot, and Y. Champagne, "Single-mode operation of a broad-area semiconductor laser with an anamorphic external cavity: Experimental and numerical results," Applied Optics, vol. 39, no. 7-36, pp. 6806-6813, 2000.

[9] S. Wolff and H. Fouckhardt, "Intracavity stabilization of broad area lasers by structured delayed optical feedback," Optics Express, vol. 7, no. 6, pp. 222-227, 2000.

[10] S. Wolff, A. Rodionov, V. E. Sherstobitov, and H. Fouckhardt, "Fourier-optical transverse mode selection in external-cavity broad-area lasers: experimental and numerical results," IEEE Journal of Quantum Electronics, vol. 39, no. 3, pp. 448-458, 2003.

[11] T. Heil, I. Fischer, and W. Elsäßer, "Coexistence of low-frequency fluctuations and stable emission on a single high-gain mode in semiconductor lasers with external optical feedback," Physical Review A: Atomic, Molecular, and Optical Physics, vol. 58, no. 4, pp. R2672-R2675, 1998.

[12] F. Rogister, P. Mégret, O. Deparis, and M. Blondel, "Coexistence of in-phase and out-of-phase dynamics in a multimode external-cavity laser diode operating in the low-frequency fluctuations regime," Physical Review A: Atomic, Molecular, and Optical Physics, vol. 62, no. 6, Article ID 061803, 4 pages, 2000.

[13] J. M. Buld, J. García-Ojalvo, M. C. Torrent, and J. M. Buldú, "Delay-induced resonances in an optical system with feedback," Physical Review E, vol. 69, Article ID 046207, 2004.

[14] S. K. Mandre, I. Fischer, and W. Elsäßer, "Spatiotemporal emission dynamics of a broad-area semiconductor laser in an external cavity: Stabilization and feedback-induced instabilities," Optics Communications, vol. 244, no. 1-6, pp. 355-365, 2005.

[15] S. Wolff, C. Doering, A. Rodionov, V. E. Sherstobitov, and H. Fouckhardt, "Self-pulsation in broad area lasers with transverse-mode selective feedback," Optics Communications, vol. 265, no. 2, pp. 642-648, 2006.

[16] J. Eichler and H. J. Eichler, Laser, Springer, Berlin, Germany, 2003.

[17] J. P. van der Ziel, "Active mode locking of double heterostructure lasers in an external cavity," Journal of Applied Physics, vol. 52, no. 7, pp. 4435-4446, 1981.

[18] Y. Silberberg, P. W. Smith, D. J. Eilenberger, D. A. B. Miller, A. C. Gossard, and W. Weigmann, "Passive mode locking of a semiconductor diode laser," Optics Letters, vol. 9, no. 11, pp. 507-509, 1984.

[19] J. E. Bowers, P. A. Morton, A. Mar, and S. W. Corzine, "Actively mode-locked semiconductor lasers," IEEE Journal of Quantum Electronics, vol. 25, no. 6, pp. 1426-1439, 1989.

[20] S. Gee and J. E. Bowers, "Ultraviolet picosecond optical pulse generation from a mode-locked InGaN laser diode," Applied Physics Letters, vol. 79, no. 13, pp. 1951-1952, 2001.

[21] L. E. Adams, E. S. Kintzer, M. Ramaswamy, J. G. Fujimoto, U. Keller, and M. T. Asom, "Mode locking of a broad-area semiconductor laser with a multiple-quantum-well saturable absorber," Optics Letters, vol. 18, no. 22, pp. 1940-1942, 1993.

[22] J. Kaiser, I. Fischer, W. Elsäßer, E. Gehrig, and O. Hess, "Modelocking in broad-area semiconductor lasers enhanced by picosecond-pulse injection," IEEE Journal on Selected Topics in Quantum Electronics, vol. 10, no. 5, pp. 968-973, 2004.

[23] J. Kaiser, I. Fischer, and W. Elsässer, "Mode locking of lateral modes in broad-area semiconductor lasers by subharmonic optical pulse injection," Applied Physics Letters, vol. 88, no. 10, Article ID 101110, 2006. 

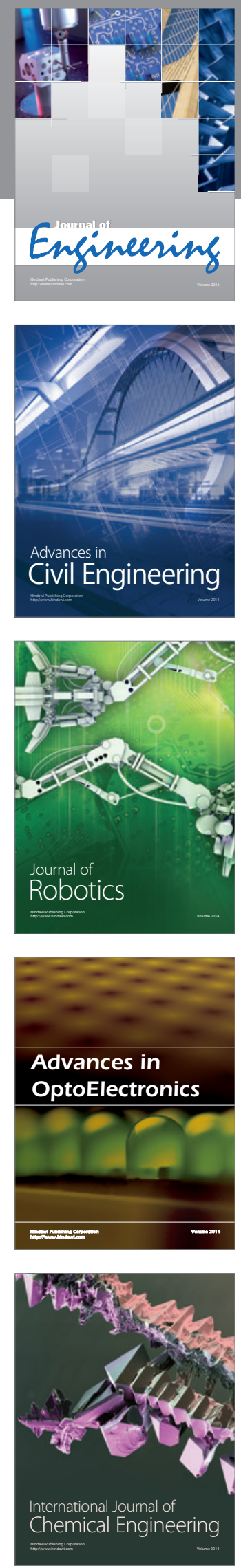

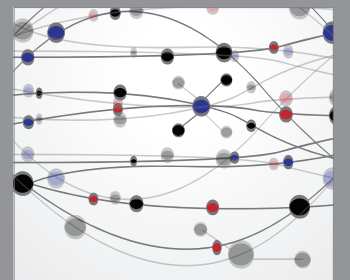

The Scientific World Journal
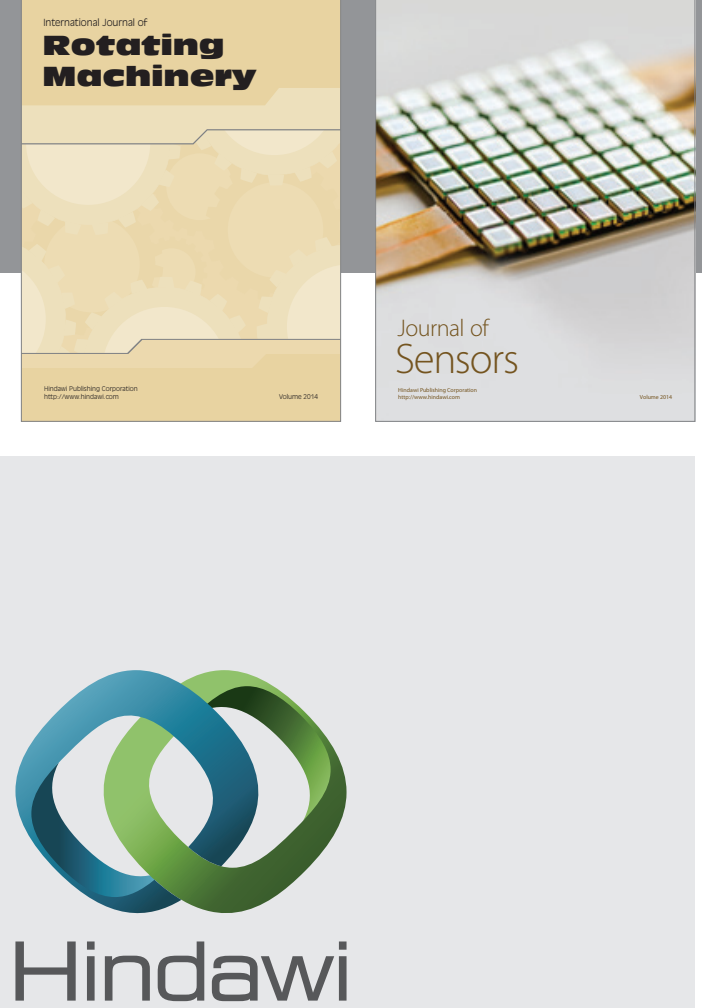

Submit your manuscripts at http://www.hindawi.com
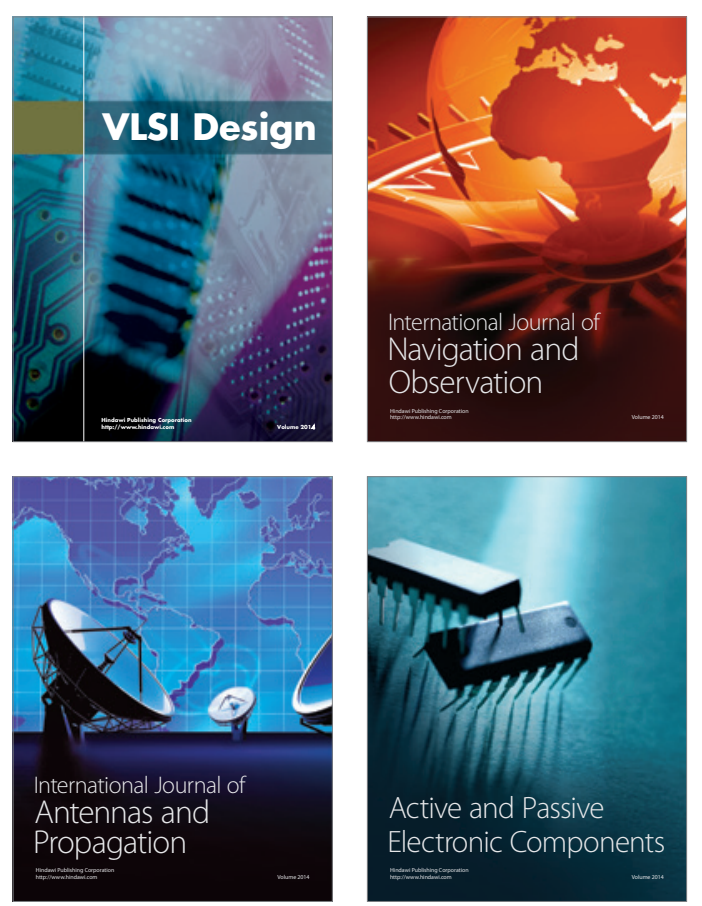
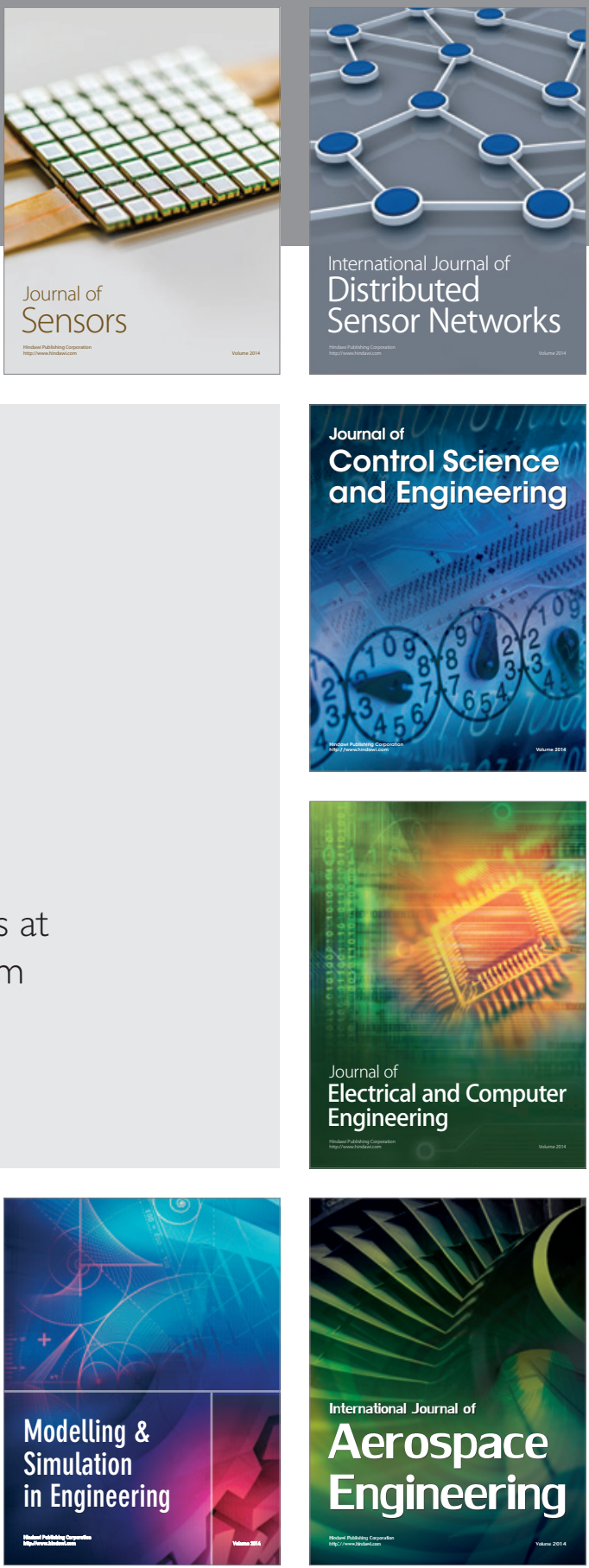

Journal of

Control Science

and Engineering
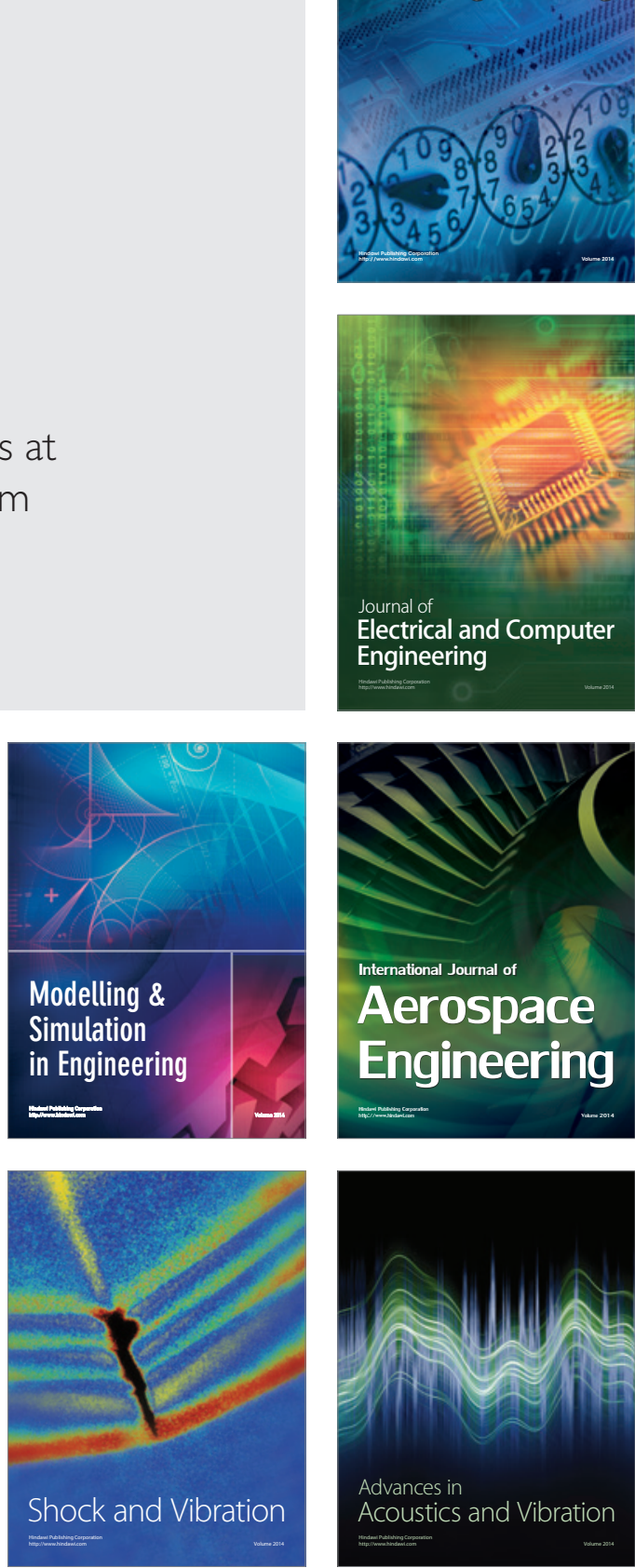